EDITORIAL

\title{
Melanocytic marker expression and TSC alterations/TFE3 fusions in uterine PEComas
}

(c) The Author(s), under exclusive licence to United States \& Canadian Academy of Pathology 2022

Modern Pathology (2022) 35:449-450; https://doi.org/10.1038/ s41379-021-01004-4

PEComas are "perivascular epithelioid cell" tumors, the origin of which is uncertain. Terms that have been used for these and related tumors are epithelioid angiomyolipoma, clear cell sugar tumor, clear cell myomelanocytic tumor, and abdominopelvic sarcoma of perivascular epithelioid cells. Furthermore, they may be associated with or resemble lymphangioleiomyomatosis and, rarely, may be a manifestation of tuberous sclerosis. PEComas can arise in many sites, but this editorial will focus on those that arise in the female genital tract, particularly the uterus. Selected references will be reviewed, but the following publication in this edition of the Journal will be highlighted: "Uterine PEComas: correlation between melanocytic marker expression and TSC alterations/TFE3 fusions" ${ }^{\prime 1}$.

To my knowledge, the first series of uterine PEComas was reported by Vang and Kempson in $2002^{2}$. They identified two types of PEComas and concluded that PEComa represented a subset of HMB-45-positive epithelioid mesenchymal tumors of the uterus with an uncertain relationship to pure smooth muscle tumors. Group A tumors frequently had clear cells with intense HMB45 labeling and less muscle differentiation and exhibiting permeative myometrial invasion, while Group B tumors had more eosinophilic cells, more smooth muscle differentiation, and less HMB45 labeling and a lesser degree of permeative myoinvasion. Following this, the MD Anderson group published two papers ${ }^{3,4}$ that cast doubt on the existence of uterine PEComa because of morphologic and immunophenotypic overlap with epithelioid smooth muscle tumors, especially those with clear cells. In one interesting example, the authors described a uterine leiomyosarcoma that became positive for HMB45 in the metastasis. Years later, Nucci and colleagues reported a series of PEComas ${ }^{5}$, including guidelines for the use of melanocyte-associated markers for diagnostic confirmation and criteria for malignancy. The criteria for malignancy have been "tweaked" in several follow-up papers (reviewed in Conlon et al. $)^{6}$, but the immunohistochemical guidelines remained: tumors that resemble PEComa can be diagnosed as such when at least two melanocyte-associated markers are present. Oliva's group reported similar results ${ }^{7}$ in that all tumors were positive for HMB-45, cathepsin $\mathrm{K}$, and at least one muscle marker, with most expressing melan-A and/or MiTF. Soon after, two published papers ${ }^{8,9}$ described details of tumors that conformed to Vang and Kempson's Group A PEComas, which we now recognize as "Xp11 PEComas" or "TFE3-translocated PEComas." Their appearance and immunophenotype are substantially different from the Group B tumors, as described above, and will not be discussed further in detail.

It should be emphasized that HMB45, Melan A and MiTF expression are not specific for PEComa. At first glance, this calls into question some of Nucci's conclusions. Oliva and Soslow studied melanocyte-associated marker expression in 9 leiomyomas, 9 epithelioid smooth muscle tumors, and 68 leiomyosarcomas (unpublished data). Among leiomyomas, 1 was positive for HMB45 ( $1+$ out of 3$), 2$ were positive for Melan A $(1+$ and $2+)$ and 5 were positive for MiTF $(1+$ and $2+)$. Among epithelioid smooth muscle tumors, 5 were $\mathrm{HMB} 45+(1+$ and $2+), 5$ expressed Melan A $(1+)$ and 2 expressed MiTF (1+). Among leiomyosarcomas, 27 were $\mathrm{HMB} 45+$ (ranging from $1+$ to $3+$ ), 22 were Melan $\mathrm{A}+$ (ranging from $1+$ to $3+$ ) and 31 of 54 were MiTF+ (ranging from $1+$ to $3+$ ). We concluded that all subcategories of smooth muscle tumors expressed HMB-45 and Melan A, with the strongest staining seen in leiomyosarcoma, but the intensity of staining was less than that reported in the literature for PEComas. MiTF reactivity may not have the same significance as expression of other markers because the majority of leiomyosarcomas were positive with this marker. These data are in keeping with previously published results from Silva's group and others.

A recently published paper calls into question the diagnostic value of myomelanocytic differentiation in tumors that are histologically high grade and ambiguous in appearance. Murali and the group from Memorial Sloan Kettering Cancer Center correlated morphologic, immunohistochemical and molecular genetic data in a group of high-grade myomelanocytic tumors ${ }^{10}$, only some of which had been diagnosed as malignant PEComas. All had expression of at least one melanocyte-like marker, usually HMB45. Among primary malignant PEComas in this study, $86 \%$ had a TSC or TFE3 alteration. The authors proposed that molecular classification of myomelanocytic tumors should take precedence over, or at least convey as much information as, immunophenotype. The premise was that "true" PEComas should have a molecular profile characteristic of that entity, regardless of the immunophenotype. Why did the authors think it was important to study phenotype-genotype correlations in this disease? It all has to do with response to agents that target the mTOR pathway. Since most classic PEComas have mTOR-activating mutations in TSC1 or 2, the term "PEComa", particularly Group B, carries with it an expectation that mTOR targeted therapy should lead to clinical response in some cases. In this study, some tumors had TSC1 or 2 mutations, others had mutations in common with other highgrade sarcomas (usually leiomyosarcoma, with $p 53$ and ATRX mutations, for example) and yet others showed evidence of bilineage differentiation in the form of leiomyosarcoma/PEComa and low-grade endometrial stromal sarcoma/PEComa. The former finding is reminiscent of Silva's description ${ }^{3}, 16$ years previously, of a leiomyosarcoma that recurred as an HMB-45 positive tumor and that would now likely be considered PEComa. The latter finding was more fully investigated in a recent publication, "TSC2-mutant uterine sarcomas with JAZF1-SUZ12 fusions demonstrate hybrid features of endometrial stromal sarcoma and PEComa and are responsive to mTOR inhibition"11.

Bennett and Oliva's paper in this edition of the Journal ${ }^{1}$ presents additional interesting results that pertain to non-ambiguous 
PEComas. The data support Nucci's recommendations for melanocyte marker positivity ${ }^{5}$, but the immunohistochemical scores were higher in the Bennett/Oliva paper. Every case showed 2-3+ (out of 3) labeling for HMB45; all but 5 expressed Melan-A (1-3+) and MiTF (also 1-3+). A few of the cases presented also support Murali's point of view ${ }^{10}$. Seventeen of 19 cases had TSC1 or TSC2 abnormalities that included not only mutations but also copy number loss and rearrangements. Four of six malignant PEComas had either TSC1 or 2 abnormalities along with $p 53$ and/or ATRX mutation, representing an association between a malignant phenotype and acquisition of mutations more commonly found in high-grade uterine sarcomas. One of the tumors studied had spindled and epithelioid components that harbored different genotypes and immunophenotypes, leiomyosarcoma-like in spindled components and PEComa-like in epithelioid components. Fusion gene analysis for JAZF1, for example, would have made this publication even more compelling. Figure 4 in this paper presents a potentially powerful diagnostic algorithm.

Several issues must now be considered: (1) Does expression of HMB45 and another related marker equate to a diagnosis of PEComa, irrespective of morphology?; (2) If not, what are the morphological criteria for PEComa?; (3) What is the value of genotyping?; (4) is uterine PEComa one entity, or several? I think it is clear from historical data that HMB45 staining alone is insufficient for a PEComa diagnosis, although when staining is diffuse and another melanocyte-associated marker is positive, the diagnosis is more likely. TSC1/2 abnormalities and TFE3 translocations are not restricted to PEComa (translocation-associated renal cell carcinoma and alveolar soft part sarcoma, for example) but are supportive of that diagnosis when the morphology and immunophenotype fit. Be aware, however, that some tumors have a PEComa appearance in which the aforementioned abnormalities are not present. When comparing the Murali paper ${ }^{10}$ with the paper by Bennett and Oliva1, it becomes clear that the preanalytical variable (i.e. how sure are you that the tumor you're studying is a PEComa?) is, perhaps, as important as the immunohistochemical and molecular phenotype.

So, what are the proper morphologic criteria for PEComa? Despite many papers describing this entity, there is still a general lack of familiarity with the appearance of PEComa, especially when malignant. Briefly, PEComas tend to show epithelioid cells arranged in vague nests surrounded by delicate vasculature. Many variations on this theme have been described ${ }^{5}$. Since it may take a specialist to diagnose this tumor accurately, one must consider diagnostic interventions that could lead to accurate diagnosis in the hands of a non-specialist, such as melanocyte-associated marker expression and a characteristic genotype. Furthermore, the identification of TSC $1 / 2$ abnormality predicts mTOR pathway activation and the possibility of response to agents that target this pathway. Lastly, there may be at least three kinds of uterine PEComas: de novo tumors with $T S C 1 / 2$ or related abnormalities; de novo tumors with TFE3 fusion; and "hybrid" PEComas with a component of either leiomyoma/sarcoma or low-grade endometrial stromal sarcoma. It is still uncertain whether the latter tumors should be classified primarily as PEComas with divergent differentiation or leiomyosarcomas or endometrial stromal sarcomas with TSC mutations. In conclusion, all the literature cited here is absolutely valid, but the work of Bennet and Oliva ${ }^{1}$ synthesizes seemingly disparate data expertly and presents a cogent, thorough and applicable examination of uterine PEComa.

\author{
Robert A. Soslow (D) ${ }^{1 凶}$ \\ ${ }^{1}$ Department of Pathology, Memorial Sloan Kettering Cancer Center, \\ New York, NY, USA. ${ }^{\bowtie}$ email: soslowr@mskcc.org
}

\section{REFERENCES}

1. Bennett J. A. et al. Uterine PEComas: correlation between melanocytic marker expression and TSC alterations/TFE3 fusions. Mod. Pathol. https://doi.org/10.1038/ s41379-021-00855-1 (2021). Online ahead of print.

2. Vang, R. \& Kempson, R. L. Perivascular epithelioid cell tumor ('PEComa') of the uterus: a subset of HMB-45-positive epithelioid mesenchymal neoplasms with an uncertain relationship to pure smooth muscle tumors. Am. J. Surg. Pathol. 26, 1-13 (2002).

3. Silva, E. G., Bodurka, D. C., Scouros, M. A. \& Ayala, A. A uterine leiomyosarcoma that became positive for HMB45 in the metastasis. Ann. Diagn. Pathol. 9, 43-45 (2005).

4. Silva, E. G., Deavers, M. T., Bodurka, D. C. \& Malpica, A. Uterine epithelioid leiomyosarcomas with clear cells: reactivity with $\mathrm{HMB}-45$ and the concept of PEComa. Am. J. Surg. Pathol. 28, 244-249 (2004).

5. Schoolmeester, J. K. et al. Perivascular epithelioid cell neoplasm (PEComa) of the gynecologic tract: clinicopathologic and immunohistochemical characterization of 16 cases. Am. J. Surg. Pathol. 38, 176-188 (2014).

6. Conlon, N., Soslow, R. A. \& Murali, R. Perivascular epithelioid tumours (PEComas) of the gynaecological tract. J. Clin. Pathol. 68, 418-426 (2015).

7. Bennett, J. A. et al. Uterine PEComas: a morphologic, immunohistochemical, andmolecular analysis of 32 tumors. Am. J. Surg. Pathol. 42, 1370-1383 (2018).

8. Agaram, N. P. et al. Dichotomy of Genetic Abnormalities in PEComas With Therapeutic Implications. Am. J. Surg. Pathol. 39, 813-825 (2015).

9. Schoolmeester, J. K. et al. TFE3 translocation-associated perivascular epithelioid cell neoplasm (PEComa) of the gynecologic tract: morphology, immunophenotype, differential diagnosis. Am. J. Surg. Pathol. 39, 394-404 (2015).

10. Selenica, P. et al. Genomic profiling aids classification of diagnostically challenging uterine mesenchymal tumors with myomelanocytic differentiation. Am. J. Surg. Pathol. 45, 77-92 (2021).

11. Chiang $\mathrm{S}$. et al. TSC2-mutant uterine sarcomas with JAZF1-SUZ12 fusions demonstrate hybrid features of endometrial stromal sarcoma and PEComa and are responsive to mTOR inhibition. Mod. Pathol. https://doi.org/10.1038/s41379021-00922-7 (2021).

\section{ACKNOWLEDGEMENTS}

I thank Dr. Sarah Chiang for her helpful review of this manuscript.

\section{FUNDING}

R.A.S. reports the following, outside the submitted work: personal fees from Ebix/ Oakstone (preparation of recorded lectures), Cambridge University Press (royalties), Springer Publishers (royalties), and Roche (lecture).

\section{COMPETING INTERESTS}

The author declares no competing interests.

\section{ADDITIONAL INFORMATION}

Correspondence and requests for materials should be addressed to Robert A. Soslow.

Reprints and permission information is available at http://www.nature.com/reprints

Publisher's note Springer Nature remains neutral with regard to jurisdictional claims in published maps and institutional affiliations. 